This is the preprint of the paper

Title: Delayed tax revenues in a class-struggle model of growth cycle

Authors: Luigi De Cesare and Mario Sportelli

Published in Communications in Nonlinear Science and Numerical Simulation, 651, 2018, 260-271

https://doi.org/10.1016/j.cnsns.2018.05.025

\title{
Delayed tax revenues in a class-struggle model of growth cycle
}

\author{
Luigi De Cesare ${ }^{\mathrm{a}}$, Mario Sportelli ${ }^{\mathrm{b}, *}$ \\ ${ }^{a}$ Dipartimento di Economia, Università di Foggia. Largo Papa Giovanni Paolo II, 1 - \\ I-71121 Foggia (Italy) \\ ${ }^{b}$ Dipartimento di Matematica, Università di Bari. Via Orabona, 4 - I-70125 Bari (Italy)
}

Abstract

In this paper, we investigate the impact of delayed tax revenues on the outcomes of fiscal policy. The analytical framework is the Goodwin growth cycle model, which is founded on the Volterra predator-prey equations. We study the dynamic behavior of the system analytically proving the existence of Hopf bifurcations, which may be supercritical and subcritical. In the numerical simulations, which follows the qualitative analysis, it is shown that, given the degree of competition in the markets, fiscal policy purposes may become consistent with their real outcomes only if policy makers are able to control the delay in the structure of the tax system. Nevertheless, there are in the system elements out of the control of the policy makers. These elements imply the possibility to make partially ineffectual the stabilization policy, because of the risk to overcome the minimum public expenditure able to stabilize the system.

Keywords: Growth cycle, Hopf bifurcation, Limit cycle.

\footnotetext{
* Corresponding author

Email addresses: luigi.decesare@unifg.it (Luigi De Cesare), mario.sportelli@uniba.it (Mario Sportelli)
} 


\section{Introduction}

In 1982, the pioneering work by Wolfstetter investigated the impact of fiscal policy in the Goodwin 1967 growth cycle model, whose analytical foundations are the well-known Volterras (1931) predator-prey equations.

Retaining the simple assumptions of Goodwin's model, Wolfstetter showed that the introduction of public sector implied a globally asymptotic stable equilibrium in the case of procyclical fiscal policy (neoclassical choice), and an unstable equilibrium in the case of a countercyclical policy rule (Keynesian choice). Furthermore, by adding two new assumptions in the model ${ }^{1}$, Wolfstetter proved that the system could be made locally stable independently of the kind of policy adopted by the government. Stability was only concerned with the strength of public expenditure.

In contrast with the Wolfstetter conclusions, Takamasu (1995) proved that only the Keynesian policy rule was able to stabilize the Goodwin cycle, but if a delay in the government decisions exists, then the model could generate chaotic economic fluctuations and no regular cycle.

In the last decade, the economic literature on stabilization policy has been enriched with many contributions dealing with the impact of fiscal policy lags on income adjustment processes. These contributions prevalently explored the effect of delays linked with the political process governing the public purchase decisions and the actual expenditures (e.g. Asada and Yoshida (2001),

\footnotetext{
${ }^{1}$ The first assumption deals with the labor market reaction to the inflation rate; the second concerns the degree of capacity utilization.
} 
Xiaofeng et al. (2005), Yoshida and Asada (2007), Matsumoto (2008)). Other contributions takes into account the question about the existence of collection lags in the tax system, i.e. the works by DeCesare and Sportelli (2005), Fanti and Manfredi (2007), Neamtu et al. (2007). All these works, except that by Asada and Yoshida (2007) analyzed the fiscal policy lags with reference to the intermediate run IS-LM model by Sasakura (1994). Never the dynamic consequences of delayed tax revenues have been considered in a class struggle model of business cycle like the one by Goodwin.

Here we filled up this gap. Our paper starts from the original Goodwin's model preserving its main hypotheses. By adding the public sector and assuming the existence of collection lags in the tax system, we emphasize: i) the firm's power market over the prices dynamics; ii) the bargaining power of workers aiming to recover the purchasing power of wages stolen from inflation. These assumptions allow us to show that the system stability requires various sets of conditions pertaining to the length of the delay and the degree of competition in the markets. For example, when the market power of firms and the relative force of workers are high enough, the minimum of the intensity of public expenditures able to achieve the stability condition may be decreasing if the time delay of the tax revenues increases. This means that, given the degree of competition in the markets, fiscal policy purposes may become consistent with their real outcomes only if policy makers have the tools suitable for the control of the delays characterizing the structure of the tax system together with a careful gage of the strength of public expenditure. Furthermore, the policy makers always have to pay attention to the degree of competition in the labor market to avoid the risk to overcome the 
minimum public expenditure able to stabilize the system.

We organized the paper as follows. Section 2 contains the formal description of the model. Section 3 is devoted to the qualitative study of the dynamic behavior of the system. Section 4 provides the numerical simulations together with the necessary economic comments. Section 5 concludes.

\section{Assumptions of the model}

Here we share all the basic assumptions of Goodwin's model:

H.1 Technical progress (neutral in the sense of Harrod) grows at a constant rate $\alpha$ :

$$
\frac{Y_{t}}{L_{t}}=a_{t}=\alpha_{0} e^{\alpha t}
$$

where $Y_{t}$ is the real output, $L_{t}$ the units of labor and $a_{t}$ the labor productivity.

H.2 Labor force $N_{t}$ grows at a constant rate $\beta$ :

$$
N_{t}=N_{0} e^{\beta t}
$$

H.3 There are only two inputs, labor and capital, both homogeneous and not sector specific.

The following assumptions are either original or borrowed from other generalizations of Goodwin's model:

H.4 Workers spend all their income in consumptions. Therefore, the aggregate saving $S_{t}$ comes from capitalist's income only. 
H.5 The price level desired by capitalists $p_{D_{t}}$ is defined as a constant markup over average costs:

$$
p_{D_{t}}=m \frac{w_{t}}{a_{t}}
$$

where $w_{t}$ is the money wage rate and $m$ the unit markup fixed in such a way to allow achievement of a profit rate over invested capital satisfying capitalist expectations.

H.6 Given the worker's bargaining power, the price $\left(p_{t}\right)$ dynamics (i.e. the inflation rate $\dot{p} / p_{t}=\pi_{t}$ ) is controlled by capitalists and represents a tool for income recovery for this class. Therefore, we set $\pi_{t}=h \dot{p}_{D} / p_{D_{t}}$, which becomes, after logarithmic differentiation of equation (1),

$$
\pi_{t}=h\left(\frac{\dot{w}}{w_{t}}-\alpha\right)
$$

where $0<h \leq 1$ denotes the elasticity of prices with respect to labor cost variations. We assume that this parameter depends on the market power in the industry.

H.7 Conventionally, as in Goodwin, we denote with $\nu_{t}=L_{t} / N_{t}$ the employment rate and with $u_{t}=w_{t} L_{t} /\left(p_{t} Y_{t}\right)=w_{t} /\left(p_{t} a_{t}\right)$ the labor income share.

H.8 The tax rate is different for workers and capitalists. Specifically, $\delta_{w}$ is the payroll tax rate and $\delta_{k}$ the tax rate on profits $\left(0<\delta_{w}<\delta_{k}<\right.$ 1). Furthermore, we assumed the existence of a finite lag between the accrual and the payment of taxes on the industry's profits. It follows that, at time $t$, the government tax revenues $(T)$ are:

$$
T_{t}=\delta_{w} u_{t} Y_{t}+\delta_{k}\left(1-\delta_{\tau}\right) Y_{\tau}
$$


where $\tau=t-\theta$ with $\theta$ a finite time $\operatorname{lag}^{2}$.

H.9 Like Yoshida and Asada (2007, p. 447), the expectations formation process of the price inflation follows a simple adaptive expectations hypothesis:

$$
\dot{\pi}^{e}=\eta\left(\pi_{t}-\pi_{t}^{e}\right)
$$

where $\pi^{e}$ is the expected inflation rate and $\eta>0$ the speed of $\pi^{e}$ adjustment to $\pi$ levels.

H.10 The adjustment process of money wage follows a standard Phillips (1958) curve, where we add a term reflecting the workers bargaining power. It allows this class to recover the purchasing power of wages stolen from inflation. Therefore, we set:

$$
\frac{\dot{w}}{w}=-\gamma+\frac{\rho}{1-\nu_{t}}+\xi \frac{u_{t}}{1-u_{t}} \pi_{t}^{e} \quad(\gamma, \rho, \xi>0)
$$

where $\xi \frac{u_{t}}{1-u_{t}}$ is a variable coefficient explaining the relative force of trade unions. We assumed that coefficient depending on the distribution of income, because, given $\pi_{t}^{e}$, the greater $\xi \frac{u_{t}}{1-u_{t}}$ is, the higher will the wage increase be and viceversa. In fact, a high wage share allows trade unions to take victorious strike actions against capitalists.

H.11 The government spending policy includes two components. The first is a regular expenditure that equals the tax revenues; the second is a discretionary expenditure proportional to the current real income.

\footnotetext{
${ }^{2}$ We have to point out that the wage tax rate has no role in the dynamic of the income growth rate, because, by assumption (H.4), workers do not save.
} 
Therefore, we set:

$$
G_{t}=T_{t}+\mu\left(\nu^{*}-\nu_{t}\right) Y_{t}
$$

where $\mu>0$ implies a counter-cyclical policy rule, while $\mu<0$ implies a pro-cyclical policy rule (Wolfstetter, 1982).

H.12 The investment function in our model is complied with a Kaldor (1961) "stylized fact" indicating the positive correlation between the share of profits in national income and the share of investment in output. Therefore, we can write:

$$
I_{t}=k\left(1-u_{t}\right) Y_{t}
$$

where $k>0$ is the propensity to invest.

H.13 According to the Keynesian tradition, changes in the aggregate output are proportional to the excess demand in the goods market. Therefore,

$$
\dot{Y}=\epsilon\left(I_{t}+G_{t}-T_{t}-S_{t}\right) \Leftrightarrow \frac{\dot{Y}}{Y_{t}}=\epsilon \frac{I_{t}+G_{t}-T_{t}-S_{t}}{Y_{t}}
$$

Taking into account equations (4) and (5), since $\frac{T_{t}}{Y_{t}}=\delta_{w} u_{t}+\delta_{k}\left(1-u_{\tau}\right) \frac{Y_{\tau}}{Y_{t}}$ and, thanks to H.4, $\frac{S_{t}}{Y_{t}}=s_{k}\left(1-u_{t}\right)-\delta_{k}\left(1-u_{\tau}\right) \frac{Y_{\tau}}{Y_{t}}$, where $0<s_{k}<1$ is the propensity to save of capitalists, equation (6) becomes,

$$
\frac{\dot{Y}}{Y_{t}}=\epsilon\left\{k\left(1-u_{t}\right)+\mu\left(\nu^{*}-\nu\right)-\left[s_{k}\left(1-u_{t}\right)-\delta_{k}\left(1-u_{\tau}\right)\right] \frac{Y_{\tau}}{Y_{t}}\right\}
$$

Looking at H.1 and H.2, the $Y_{\tau} / Y_{t}$ ratio can be written as follows

$$
\frac{Y_{\tau}}{Y_{t}}=\frac{\alpha_{0} e^{\alpha \tau} L_{\tau}}{\alpha_{0} e^{\alpha t} L_{t}}=\frac{e^{\alpha \tau} N_{0} e^{\beta \tau} \nu_{\tau}}{e^{\alpha t} N_{0} e^{\beta t} \nu_{t}}=\frac{\nu_{\tau}}{e^{(\alpha+\beta)(t-\tau)} \nu_{t}}
$$


Therefore, after some rearrangement, the growth rate of income becomes:

$$
\frac{\dot{Y}}{Y_{t}}=\epsilon\left\{\left(k-s_{k}\right)\left(1-u_{t}\right)+\mu\left(\nu^{*}-\nu_{t}\right)+s_{k} \delta_{k}\left(1-u_{\tau}\right) \frac{\nu_{\tau}}{e^{(\alpha+\beta)(t-\tau)} \nu_{t}}\right\}
$$

where we set $k-s_{k}>0$ according to the Kaldor (1940) tradition ${ }^{3}$.

Given the hypothesis H.7, logarithmic differentiation of $\nu_{t}$ yields $\frac{\dot{\nu}}{\nu_{t}}=$ $\frac{\dot{Y}}{Y_{t}}-(\alpha+\beta)$, where, the substitution of equation (7) allows us to write:

$\dot{\nu}=\left\{\epsilon\left[\left(k-s_{k}\right)\left(1-u_{t}\right)+\mu\left(\nu^{*}-\nu_{t}\right)+s_{k} \delta_{k}\left(1-u_{\tau}\right) \frac{\nu_{\tau}}{e^{(\alpha+\beta)(t-\tau)} \nu_{t}}\right]-(\alpha+\beta)\right\} \nu_{t}$

Logarithmic differentiation of $u_{t}$ yields $\frac{\dot{u}}{u_{t}}=\frac{\dot{w}}{w_{t}}-\pi_{t}-\alpha$ where the substitution of equations (2) and (3) allows us to write, after some tedious rearrangement:

$$
\dot{u}=\left\{(1-h)\left[\frac{\rho}{1-\nu_{t}}+\xi \frac{u_{t}}{1-u_{t}} \pi_{t}^{e}-(\alpha+\gamma)\right]\right\} u_{t}
$$

Finally, after the appropriate substitutions, the hypothesis H.9 yields the following equation:

$$
\dot{\pi}^{e}=\eta\left\{h\left[\frac{\rho}{1-\nu_{t}}-(\alpha+\gamma)\right]+\left(h \xi \frac{u_{t}}{1-u_{t}}-1\right) \pi_{t}^{e}\right\}
$$

The three equations (8), (9) and (10) make up a time delay system of differential equations (DDEs) we shall study in the next section.

\section{Qualitative analysis of the model}

Without loss of generality we set $\epsilon=1$. In compact form, our dynamical system is

\footnotetext{
${ }^{3}$ In his 1940 business cycle model, Kaldor assumed that the propensity to invest was always greater than the propensity to save.
} 


$$
\left\{\begin{array}{l}
\dot{\nu}=\left(\frac{\dot{Y}}{Y_{t}}-(\alpha+\beta)\right) \nu_{t} \\
\dot{u}=(1-h)\left(\frac{\dot{w}}{w_{t}}-\alpha\right) u_{t} \\
\dot{\pi}^{e}=\eta\left[h\left(\frac{\dot{w}}{w_{t}}-\alpha\right)-\pi_{t}^{e}\right]
\end{array}\right.
$$

\subsection{The case $\theta=0$}

In this section, we examine the case $\theta=0$. The growth rate of income (7) becomes

$$
\frac{\dot{Y}}{Y_{t}}=\left(k-s_{k}\left(1-\delta_{k}\right)\right)\left(1-u_{t}\right)+\mu\left(\nu^{*}-\nu_{t}\right) .
$$

We suppose that

$$
\rho<\alpha+\gamma, \quad \alpha+\beta<k-s_{k}\left(1-\delta_{k}\right) .
$$

It is easy to show that system (11) has three equilibrium solutions
A) $\nu_{1}=0, \quad u_{1}=0, \quad \pi_{1}^{e}=h[\rho-(\alpha+\gamma)]$
B) $\nu_{2}=\nu^{*}+\nu^{B}, \quad u_{2}=0, \quad \pi_{2}^{e}=h\left(\frac{\rho}{1-\left(\nu^{*}+\nu^{B}\right)}-(\alpha+\gamma)\right)$
C) $\nu_{3}=\nu^{*}, \quad u_{3}=u^{*}, \quad \pi_{3}^{e}=0$

where $\nu^{*}=1-\frac{\rho}{\alpha+\gamma}, u^{*}=1-\frac{\alpha+\beta}{k-s_{k}\left(1-\delta_{k}\right)}$ and $\nu^{B}=\frac{k-s_{k}\left(1-\delta_{k}\right)-(\alpha+\beta)}{\mu}$. The inequalities (12), imply that

$$
0<v^{*}<1, \quad 0<u^{*}<1, \quad \nu^{B}>0
$$

moreover if $\nu^{*}+\nu^{B}<1$ that is

$$
\nu^{B}<\frac{\rho}{\alpha+\gamma}
$$


we have

$$
\frac{\rho}{1-\left(\nu^{*}+\nu^{B}\right)}-(\alpha+\gamma)=\rho\left(\frac{1}{\frac{\rho}{\alpha+\gamma}-\nu^{B}}-\frac{1}{\frac{\rho}{\alpha+\gamma}}\right)>0
$$

As usual, we investigate the local dynamics of system (11) analytically by means of the linear approximation method. Expanding the system (11) in a Taylor series around an equilibrium point $\left(\nu_{i}, u_{i}, \pi_{i}^{e}\right), i=1,2,3$, and neglecting the terms of higher order than the first order, we have the following linear approximation:

$$
\left(\begin{array}{l}
\dot{\nu} \\
\dot{u} \\
\dot{\pi}^{e}
\end{array}\right)=\left(\begin{array}{ccc}
\frac{\dot{Y}}{Y_{t}}-(\alpha+\beta)-\mu \nu_{i} & -\nu_{i}\left(k-s_{k}\left(1-\delta_{k}\right)\right) & 0 \\
(1-h) u_{i} \frac{\partial}{\partial \nu} \frac{\dot{w}}{w_{t}} & (1-h)\left[\left(\frac{\dot{w}}{w_{t}}-\alpha\right)+u_{i} \frac{\partial}{\partial u} \frac{\dot{w}}{w_{t}}\right] & (1-h) u_{i} \frac{\partial}{\partial \pi^{e}} \frac{\dot{w}}{w_{t}} \\
\eta h \frac{\partial}{\partial \nu} \frac{\dot{w}}{w_{t}} & \eta h \frac{\partial}{\partial u} \frac{\dot{w}}{w_{t}} & \eta\left(h \frac{\partial}{\partial \pi^{e}} \frac{\dot{w}}{w_{t}}-1\right)
\end{array}\right)\left(\begin{array}{l}
\nu_{t}-\nu_{i} \\
u_{t}-u_{i} \\
\pi_{t}^{e}-\pi_{i}^{e}
\end{array}\right)
$$

where $\frac{\partial}{\partial \nu} \frac{\dot{w}}{w_{t}}=\frac{\rho}{\left(1-\nu_{i}\right)^{2}}, \frac{\partial}{\partial u} \frac{\dot{w}}{w_{t}}=\xi \frac{\pi_{i}^{e}}{\left(1-u_{i}\right)^{2}}$ and $\frac{\partial}{\partial \pi^{e}} \frac{\dot{w}}{w_{t}}=\xi \frac{u_{i}}{1-u_{i}}, i=$ $1,2,3$.

The Jacobian of the system (11) at the equilibrium point A) is a triangular matrix

$$
\left(\begin{array}{ccc}
\mu \nu^{*}+k-s_{k}\left(1-\delta_{k}\right)-(\alpha+\beta) & 0 & 0 \\
0 & (1-h)(\rho-(\alpha+\gamma)) & 0 \\
\eta h \frac{\partial}{\partial \nu} \frac{\dot{w}}{w_{t}} & \eta h \frac{\partial}{\partial u} \frac{\dot{w}}{w_{t}} & -\eta
\end{array}\right)
$$

so that the eigenvalues are $\lambda_{1}=\mu \nu^{*}+k-s_{k}\left(1-\delta_{k}\right)-(\alpha+\beta)>0, \lambda_{2}=$ $(1-h)[\rho-(\alpha+\gamma)]<0$ and $\lambda_{3}=-\eta<0$. This equilibrium is locally unstable.

The Jacobian of the system (11) at the equilibrium point B) is

$$
\left(\begin{array}{ccc}
-\mu\left(\nu^{*}+\nu^{B}\right) & -\left(\nu^{*}+\nu^{B}\right)\left(k-s_{k}\left(1-\delta_{k}\right)\right) & 0 \\
0 & (1-h)\left[\frac{\rho}{1-\left(\nu^{*}+\nu^{B}\right)}-(\alpha+\gamma)\right] & 0 \\
\eta h \frac{\partial}{\partial \nu} \frac{\dot{w}}{w_{t}} & \eta h \frac{\partial}{\partial u} \frac{\dot{w}}{w_{t}} & -\eta
\end{array}\right)
$$


Its characteristic equation is

$$
-(\lambda+\eta)\left[\lambda^{2}-\left(a_{11}+a_{22}\right) \lambda+a_{11} a_{22}\right]=0
$$

where $a_{11}=-\mu\left(\nu^{*}+\nu^{B}\right)<0$ and $a_{22}=(1-h)\left[\frac{\rho}{1-\left(\nu^{*}+\nu^{B}\right)}-(\alpha+\gamma)\right]$. If condition (13) holds, the inequality (14) implies that $a_{22}>0$. Therefore the characteristic equation (16) admits two negative real solutions and one positive real solution. It follows that the equilibrium point B) is unstable.

Furthermore, if condition (13) does not hold, the inequality (14) implies that $a_{22}<0$. In this case, the characteristic equation (16) admits three negative real solutions. It follows that the equilibrium point B) is stable, but it has no economic meaning, because $\nu_{2}=\nu^{*}+\nu^{B}>1$.

Finally, let us note that the equilibrium point B) is still unstable if

$$
\mu>\frac{\alpha+\gamma}{\rho}\left(k-s_{k}\left(1-\delta_{k}\right)-(\alpha+\beta)\right)
$$

otherwise it is stable.

We now examine the Jacobian of the system (11) at the equilibrium point C). In this case we have

$$
\left(\begin{array}{ccc}
a_{11} & a_{12} & 0 \\
a_{21} & 0 & a_{23} \\
a_{31} & 0 & a_{33}
\end{array}\right):=\left(\begin{array}{ccc}
-\mu \nu^{*} & -\nu^{*}\left(k-s_{k}\left(1-\delta_{k}\right)\right) & 0 \\
(1-h) u^{*} \frac{\rho}{\left(1-\nu^{*}\right)^{2}} & 0 & (1-h) u^{*} \xi \frac{u^{*}}{1-u^{*}} \\
\eta h \frac{\rho}{\left(1-\nu^{*}\right)^{2}} & 0 & -\eta\left(1-h \xi \frac{u^{*}}{1-u^{*}}\right)
\end{array}\right)
$$

The characteristic equation is

$$
\lambda^{3}+a_{2} \lambda^{2}+a_{1} \lambda+a_{0}=0
$$

where, by setting

$$
q=1-h \xi \frac{u^{*}}{1-u^{*}}=1-h \xi\left(\frac{k-s_{k}\left(1-\delta_{k}\right)}{\alpha+\beta}-1\right)
$$


$z=(1-h) u^{*} \frac{\rho}{\left(1-\nu^{*}\right)^{2}} \nu^{*}\left(k-s_{k}\left(1-\delta_{k}\right)\right)=(1-h) \frac{\alpha+\gamma}{\rho}(\alpha+\gamma-\rho)\left(k-s_{k}\left(1-\delta_{k}\right)\right)$,

we have

$$
\left\{\begin{array}{l}
a_{2}=-\left(a_{11}+a_{33}\right)=\mu \nu^{*}+\eta q, \\
a_{1}=-a_{12} a_{21}+a_{11} a_{33}=z+\mu \nu^{*} \eta q, \\
a_{0}=a_{12}\left(a_{21} a_{33}-a_{23} a_{31}\right)=\eta z .
\end{array}\right.
$$

Looking at the inequality $(12)$, it results $a_{0}=\eta z>0$.

It follows, according to the Routh-Hurwitz criterion, that all the solutions of the characteristic equation (17) have negative real parts if and only if

$$
a_{0}>0, \quad a_{2}>0, \quad a_{1} a_{2}-a_{0}>0 .
$$

Taking into account that

$$
a_{1} a_{2}-a_{0}=\left(\nu^{*}\right)^{2} \eta q \mu^{2}+\nu^{*}\left(\eta^{2} q^{2}+z\right) \mu+\eta z(q-1)
$$

we set the following two theorems

Theorem 1. If

$$
h \xi\left(\frac{k-s_{k}\left(1-\delta_{k}\right)}{\alpha+\beta}-1\right) \leq 1
$$

there exists $\bar{\mu}>0$ such that the equilibrium point $\left(\nu^{*}, u^{*}, 0\right)$ is

$$
\begin{array}{ll}
\text { locally unstable } & \text { if } \mu<\bar{\mu}, \\
\text { locally stable } & \text { if } \mu>\bar{\mu} .
\end{array}
$$

Proof. The Routh-Hurwitz stability criterion assures that the equilibrium point is locally stable if and only if

$$
\left\{\begin{array}{l}
f(\mu):=\nu^{*} \mu+\eta q>0 \\
g(\mu):=\left(\nu^{*}\right)^{2} \eta q \mu^{2}+\nu^{*}\left(\eta^{2} q^{2}+z\right) \mu+\eta z(q-1)>0
\end{array}\right.
$$


Let us note that hypothesis (19) implies $0 \leq q<1$. So that, if $q>0$, the second-order polynomial $g(\mu)$ has one negative root and one positive root:

$$
\mu_{1 / 2}=\frac{-\left(\eta^{2} q^{2}+z\right) \pm \sqrt{\left(\eta^{2} q^{2}-z\right)^{2}+4 \eta^{2} q z}}{2 \nu^{*} \eta q}
$$

It is easy to show that

$$
\mu_{1}=\frac{-\left(\eta^{2} q^{2}+z\right)-\sqrt{\left(\eta^{2} q^{2}-z\right)^{2}+4 \eta^{2} q z}}{2 \nu^{*} \eta q}<-\frac{\eta q}{\nu^{*}}<0 .
$$

Let us note that $f(\mu)>0$ if and only if $\mu \in]-\frac{\eta q}{\nu^{*}},+\infty[$ and $g(\mu)>0$ if and only if $\mu \in]-\infty, \mu_{1}[\cup] \mu_{2},+\infty[$. It follows that $f(\mu)>0$ and $g(\mu)>0$ if and only if

$$
\mu>\bar{\mu}:=\mu_{2}=\frac{-\left(\eta^{2} q^{2}+z\right)+\sqrt{\left(\eta^{2} q^{2}-z\right)^{2}+4 \eta^{2} q z}}{2 \nu^{*} \eta q}>0 .
$$

If $q=0$, then conditions (20) hold if and only if $\mu>\frac{\eta}{\nu^{*}}$.

Theorem 2. By assuming that

$$
h \xi\left(\frac{k-s_{k}\left(1-\delta_{k}\right)}{\alpha+\beta}-1\right)>1,
$$

if the following conditions hold

$$
\left(\eta^{2} q^{2}-z\right)^{2}+4 \eta^{2} q z>0 \quad \text { and } \quad \eta^{2} q^{2}<z
$$

then two positive stability switch $\bar{\mu}_{1}$ and $\bar{\mu}_{2}$ exist and the equilibrium point $\left(\nu^{*}, u^{*}, 0\right)$ is locally stable if $\left.\mu \in\right] \bar{\mu}_{1}, \bar{\mu}_{2}[$, otherwise the equilibrium is unstable.

Moreover if condition (23) does not hold, i.e.

$$
\left(\eta^{2} q^{2}-z\right)^{2}+4 \eta^{2} q z<0 \quad \text { or } \quad \eta^{2} q^{2}>z
$$

then $\left(\nu^{*}, u^{*}, 0\right)$ in locally unstable. 
Proof. According to the Routh-Hurwitz stability criterion, the equilibrium point is locally stable if and only if conditions (20) hold. In this case, from the inequality(22), we have $q<0$.

Let us suppose that inequalities $(23)$ do not hold. In this case $\left(\eta^{2} q^{2}-\right.$ $z)^{2}+4 \eta^{2} q z<0$, the second-order polynomial $g(\mu)$ has no real roots hence for all $\mu$ we have $g(\mu)<0$ and the equilibrium point is unstable.

In the case $\left(\eta^{2} q^{2}-z\right)^{2}+4 \eta^{2} q z>0, g(\mu)$ has two positive real roots given by expression (21). Let us note that $f(\mu)>0$ if and only if $\mu \in]-\frac{\eta q}{\nu^{*}},+\infty[$ and $g(\mu)>0$ iff $\mu \in] \mu_{2}, \mu_{1}\left[\right.$. Therefore, assuming that $\eta^{2} q^{2}>z$, it is easy to show that

$$
0<\mu_{2}<\mu_{1}<-\frac{\eta q}{\nu^{*}}
$$

It follows that conditions (20) have no solutions with respect to $\mu$ and the equilibrium point is unstable.

Now, let us suppose that inequalities (23) hold. In this case we can show that

$$
0<-\frac{\eta q}{\nu^{*}}<\mu_{2}<\mu_{1}
$$

hence the Routh-Hurwitz conditions (20) are satisfied for all $\mu$ in the interval ] $\bar{\mu}_{1}, \bar{\mu}_{2}\left[\right.$ where $\bar{\mu}_{1}=\mu_{2}$ and $\bar{\mu}_{2}=\mu_{1}$.

Remarks.

If $\mu<0$ the equilibrium point is always unstable.

If $h \xi$ is "small" then condition (19) holds and the equilibrium is unstable for $\mu$ "small" and stable for $\mu$ "high enough".

If $h \xi$ is not small and the condition (22) holds, then the equilibrium is always unstable. Otherwise, if conditions (23) hold a stability region exists. 


\subsubsection{Hopf Bifurcations}

Theorem 3. If hypothesis of Theorem 1 hold, then system (11) exhibits a Hopf bifurcation at $\left(\nu^{*}, u^{*}, 0\right)$ when $\mu=\bar{\mu}$ i.e. there exists a continuous family of non-constant periodic solutions of the system (11) which collapses to equilibrium point.

Proof. In order to apply the Hopf bifurcation theorem, we prove that the characteristic equation (17) has a pair of pure imaginary eigenvalues and no other eigenvalues with zero real parts when $\mu=\bar{\mu}$ and the following transversality condition holds:

$$
\left.\frac{d \operatorname{Re} \lambda(\mu)}{d \mu}\right|_{\mu=\bar{\mu}} \neq 0
$$

where $\operatorname{Re} \lambda(\mu)$ is the real part of $\lambda(\mu)$.

First of all let us note that characteristic equation (17) has a pair of imaginary roots iff $a_{1}>0$ and $a_{1} a_{2}-a_{0}=0$. In our case, from (20), we have that $a_{2}(\bar{\mu})=f\left(\mu_{2}\right)>0$ and $a_{1}(\bar{\mu}) a_{2}(\bar{\mu})-a_{0}(\bar{\mu})=g\left(\mu_{2}\right)=0$. From $a_{2}(\bar{\mu})>0$ and $a_{0}>0$, it follows that $a_{1}(\bar{\mu})>0$. Furthermore, from $a_{0}>0$, the real root of (17) is different from zero. It can be shown that the imaginary roots are $\lambda= \pm i \sqrt{a_{1}}$.

By implicit differentiation of

$$
F(\mu, \lambda):=\lambda^{3}+a_{2}(\mu) \lambda^{2}+a_{1}(\mu) \lambda+a_{0}(\mu)
$$

we have that, omitting the dependence on the variable $\mu$,

$$
\frac{d \lambda}{d \mu}=-\frac{\frac{\partial F}{\partial \mu}}{\frac{\partial F}{\partial \lambda}}=-\frac{\lambda^{2} \frac{\partial a_{2}}{\partial \mu}+\lambda \frac{\partial a_{1}}{\partial \mu}+\frac{\partial a_{0}}{\partial \mu}}{3 \lambda^{3}+2 a_{2} \lambda+a_{1}}
$$


Taking into account that $\lambda=i \sqrt{a_{1}}$, we obtain, after some calculations,

$$
\left.\frac{d \operatorname{Re} \lambda(\mu)}{d \mu}\right|_{\mu=\bar{\mu}}=\frac{\frac{\partial a_{0}}{\partial \mu}-a_{1} \frac{\partial a_{2}}{\partial \mu}-a_{2} \frac{\partial a_{1}}{\partial \mu}}{2\left(a_{1}+a_{2}^{2}\right)} .
$$

Let us note that, if $q>0$,

$$
\begin{gathered}
\frac{\partial a_{0}}{\partial \mu}-a_{1} \frac{\partial a_{2}}{\partial \mu}-a_{2} \frac{\partial a_{1}}{\partial \mu}=-\nu^{*}\left(z+2 \mu_{2} \nu^{*} \eta q+\eta^{2} q^{2}\right)= \\
=-\nu^{*} \sqrt{\left(\eta^{2} q^{2}-z\right)^{2}+4 \eta^{2} q z}<0 .
\end{gathered}
$$

If $q=0$, we have

$$
\frac{\partial a_{0}}{\partial \mu}-a_{1} \frac{\partial a_{2}}{\partial \mu}-a_{2} \frac{\partial a_{1}}{\partial \mu}=-\nu^{*} z<0
$$

Theorem 4. If hypothesis (22) and (23) of Theorem 2 hold, then the system (11) exhibits Hopf bifurcations at $\left(\nu^{*}, u^{*}, 0\right)$ when $\mu=\bar{\mu}_{1}$ and $\mu=\bar{\mu}_{2}$.

Proof. The proof is very similar to the previous one. In this case the transversality conditions are

$$
\left.\frac{d \operatorname{Re} \lambda(\mu)}{d \mu}\right|_{\mu=\bar{\mu}_{1}}<0,\left.\quad \frac{d \operatorname{Re} \lambda(\mu)}{d \mu}\right|_{\mu=\bar{\mu}_{2}}>0 .
$$

The emerging periodic solutions in previous Hopf bifurcations could be stables or unstables. In order to study the bifurcation properties, we use the first Lyapunov coefficient (see Kuznetsov, 2004 for more details). It is well known that if the first Lyapunov coefficient $l_{1}(\bar{x}, \bar{\mu})$ evaluated at the bifurcation point $\bar{x}$ is negative the limit cycle is stable (supercritical Hopf bifurcation) otherwise if $l_{1}(\bar{x}, \bar{\mu})$ is positive the limit cycle is unstable (subcritical Hopf bifurcation). If the first Lyapunov coefficient vanish at bifurcation 
point, the stability of Hopf bifurcation is determined in the same manner by second Lyapunov coefficient.

In our case, we have an autonomous system of nonlinear ordinary differential equation depending on a parameter $\mu \in \mathbb{R}$ :

$$
x^{\prime}=f(x, \mu) \quad x \in \mathbb{R}^{3}
$$

where $f$ is given by the right hand side of (11) and $x=\left(\nu-\nu^{*}, u-u^{*}, \pi^{e}\right)^{T}$. The system has an equilibrium point at the origin $\bar{x}=(0,0,0)^{T}$ which does not depend on $\mu$ and exhibit an Hopf bifurcation when $\mu=\bar{\mu}$. The Jacobian matrix $J_{0}=\left.D_{x} f\right|_{(\bar{x}, \bar{\mu})}$ has two purely imaginary complex conjugate eigenvalues, given by $\pm i \omega$, where $\omega=\sqrt{a_{1}}$. The Taylor expansion of $f$ near the origin is

$$
f(x, \bar{\mu})=J_{0} x+\frac{1}{2} B(x, x)+\frac{1}{6} C(x, x, x)+O\left(\|x\|^{4}\right)
$$

where $B$ and $C$ are respectively the second and third order terms. $B$ and $C$ are multilinear functions with components given by

$$
\begin{gathered}
B_{m}(x, y)=\left.\sum_{i=1}^{3} \sum_{j=1}^{3} \frac{\partial^{2} f_{m}(w, \bar{\mu})}{\partial w_{i} \partial w_{j}}\right|_{w=\bar{x}} x_{i} y_{j} \quad m=1,2,3 \\
C_{m}(x, y, z)=\left.\sum_{i=1}^{3} \sum_{j=1}^{3} \sum_{k=1}^{3} \frac{\partial^{3} f_{m}(w, \bar{\mu})}{\partial w_{i} \partial w_{j} \partial w_{k}}\right|_{w=\bar{x}} x_{i} y_{j} z_{k} \quad m=1,2,3
\end{gathered}
$$

Let $\mathbf{q} \in \mathbb{C}^{3}$ a complex eigenvector of Jacobian matrix $J_{0}$ corresponding to the eigenvalue $i \omega$ and let $\mathbf{p} \in \mathbb{C}^{3}$ a so called adjoint eigenvector that is an eigenvector of the transpose of $J_{0}$ corresponding to eigenvalue $-i \omega$. We choose $\mathbf{q}$ and $\mathbf{p}$ such that their inner product in $\mathbb{C}$ is equal to one. The first Lyapunov coefficient is defined by

$$
l_{1}(\bar{x}, \bar{\mu})=\frac{1}{2 \omega} \operatorname{Re}\left(T_{1}-2 T_{2}+T_{3}\right)
$$


where

$$
\begin{gathered}
T_{1}=<\mathbf{p}, C(\mathbf{q}, \mathbf{q}, \overline{\mathbf{q}})> \\
T_{2}=<\mathbf{p}, B\left(\mathbf{q}, J_{0}^{-1} B(\mathbf{q}, \overline{\mathbf{q}})\right)> \\
T_{3}=<\mathbf{p}, B\left(\overline{\mathbf{q}},\left(2 i \omega I-J_{0}\right)^{-1}, B(\mathbf{q}, \mathbf{q})\right)>
\end{gathered}
$$

and $I$ is the unit matrix, $\langle\cdot, \cdot\rangle$ denotes the inner product and $\overline{\mathbf{q}}$ is the complex conjugate of $\mathbf{q}$.

The first Lyapunov coefficient has been calculated by means of the bifurcation analysis software "DDE-BIFTOOL" which is a MATLAB numerical continuation package developed by K. Engelborghs et al. (2002).

\subsection{The case $\theta>0$}

Using the same argumentation of the case $\theta=0$, replacing the parameter $\delta_{k}$ with $\delta_{k} e^{-(\alpha+\beta) \theta}$ in inequalities (12) and in definitions of $u^{*}$ and $v^{B}$ we have formally the three equilibrium solutions A), B) and C). Hence, if $\theta>0$, the inequalities (12) become

$$
\rho<\alpha+\gamma, \quad \alpha+\beta<k-s_{k}\left(1-\delta_{k} e^{-(\alpha+\beta) \theta}\right)
$$

The values $\nu_{2}$ and $\pi_{2}^{e}$ (equilibrium point B)) grow when the delay $\theta$ increases and the value $u_{3}$ (equilibrium point $\mathrm{C}$ )) decreases when the delay $\theta$ increases.

Expanding the system (11) in a Taylor series around an equilibrium point $\left(\nu_{i}, u_{i}, \pi_{i}^{e}\right), i=1,2,3$, and neglecting the terms of higher order than the first order, we have the following linear approximation:

$$
\left(\begin{array}{c}
\dot{\nu} \\
\dot{u} \\
\dot{\pi}^{e}
\end{array}\right)=A\left(\begin{array}{c}
\nu_{t}-\nu_{i} \\
u_{t}-u_{i} \\
\pi_{t}^{e}-\pi_{i}^{e}
\end{array}\right)+B\left(\begin{array}{c}
\nu_{t-\theta}-\nu_{i} \\
u_{t-\theta}-u_{i} \\
\pi_{t-\theta}^{e}-\pi_{i}^{e}
\end{array}\right)
$$


where

$$
\begin{gathered}
\mathcal{A}=\left(\begin{array}{ccc}
\frac{\partial}{\partial \nu}\left(\nu \frac{\dot{Y}}{Y_{t}}\right)-(\alpha+\beta) & -\nu_{i}\left(k-s_{k}\right) & 0 \\
(1-h) u_{i} \frac{\partial}{\partial \nu} \frac{\dot{w}}{w_{t}} & (1-h)\left[\frac{\dot{w}}{w_{t}}-\alpha+u_{i} \frac{\partial}{\partial u} \frac{\dot{w}}{w_{t}}\right] & (1-h) u_{i} \frac{\partial}{\partial \pi^{e}} \frac{\dot{w}}{w_{t}} \\
\eta h \frac{\partial}{\partial \nu} \frac{\dot{w}}{w_{t}} & \eta h \frac{\partial}{\partial u} \frac{\dot{w}}{w_{t}} & \eta\left(h \frac{\partial}{\partial \pi^{e}} \frac{\dot{w}}{w_{t}}-1\right)
\end{array}\right) \\
\mathcal{B}=\left(\begin{array}{ccc}
s_{k} \delta_{k}\left(1-u_{i}\right) e^{-(\alpha+\beta) \theta} & -s_{k} \delta_{k} \nu_{i} e^{-(\alpha+\beta) \theta} & 0 \\
0 & 0 & 0 \\
0 & 0 & 0
\end{array}\right)
\end{gathered}
$$

To investigate linear stability we need to determine the eigenvalues of the system (25) which are the solutions of the characteristic equation

$$
\operatorname{det}\left(\lambda I-\mathcal{A}-\mathcal{B} e^{-\lambda \theta}\right)=0
$$

After some calculations, the characteristic equation at equilibrium point A) becomes

$\left[\lambda-s_{k} \delta_{k} e^{-(\alpha+\beta) \theta} e^{-\theta \lambda}-\left(k-s_{k}\right)-\mu \nu^{*}+\alpha+\beta\right][\lambda-(1-h)(\rho-(\alpha+\gamma))][\lambda+\eta]=0$.

We have two real negative eigenvalues $\lambda_{1}=-\eta$ and $\lambda_{2}=(1-h)(\rho-(\alpha+\gamma))$ and infinite complex eigenvalues given by

$$
f(\lambda):=\lambda-s_{k} \delta_{k} e^{-(\alpha+\beta) \theta} e^{-\theta \lambda}-\left(k-s_{k}\right)-\mu \nu^{*}+\alpha+\beta=0 .
$$

We note that $f(0)=-s_{k} \delta_{k} e^{-(\alpha+\beta) \theta}-\left(k-s_{k}\right)-\mu \nu^{*}+\alpha+\beta$ which, from inequality (24), is negative. Given that $\lim _{\lambda \rightarrow+\infty} f(\lambda)=+\infty$, we conclude that there exist a real positive eigenvalue, so that the equilibrium point A) is always unstable. 
The characteristic equation at equilibrium point B) becomes

$$
\left[\lambda-s_{k} \delta_{k} e^{-(\alpha+\beta) \theta} e^{-\theta \lambda}-\left(k-s_{k}\right)+2 \mu \nu_{2}-\mu \nu^{*}+\alpha+\beta\right]\left[\lambda-(1-h)\left(\frac{\rho}{1-\nu_{2}}-(\alpha+\gamma)\right)\right][\lambda+\eta]=0 .
$$

In this case we have a real negative eigenvalue $\lambda_{1}=-\eta$ and a real eigenvalue $\lambda_{2}=(1-h)\left(\frac{\rho}{1-\nu_{2}}-(\alpha+\gamma)\right)$. If we suppose that $\nu_{2}<1$, from inequality (14), $\lambda_{2}$ is positive. Hence the equilibrium point B) is unstable.

Substituting the equilibrium point $\mathrm{C})\left(\nu^{*}, u^{*}, 0\right)$ in the Jacobian matrix (26), we have

$$
\mathcal{A}=\left(\begin{array}{ccc}
\left(k-s_{k}\right)\left(1-u^{*}\right)-\mu \nu^{*}-(\alpha+\beta) & -\nu^{*}\left(k-s_{k}\right) & 0 \\
(1-h) u^{*} \frac{\rho}{\left(1-\nu^{*}\right)^{2}} & 0 & (1-h) u^{*} \xi \frac{u^{*}}{1-u^{*}} \\
\eta h \frac{\rho}{\left(1-\nu^{*}\right)^{2}} & 0 & \eta\left(h \xi \frac{u^{*}}{1-u^{*}}-1\right)
\end{array}\right)
$$

The characteristic equation at equilibrium point $\mathrm{C}$ ) is

$$
\begin{gathered}
\lambda^{3}-\left(a_{11}+a_{33}\right) \lambda^{2}+\left(a_{11} a_{33}-a_{12} a_{21}\right) \lambda+a_{12}\left(a_{21} a_{33}-a_{23} a_{31}\right)+ \\
\left(-b_{11} \lambda^{2}+\left(a_{33} b_{11}-a_{21} b_{12}\right) \lambda+b_{12}\left(a_{21} a_{33}-a_{23} a_{31}\right)\right) e^{-\theta \lambda}=0
\end{gathered}
$$

where $a_{i j}$ and $b_{i j}$ are respectively the entries of matrix $\mathcal{A}$ and $\mathcal{B}$. We note that $a_{11}, a_{12}$ and $b_{12}$ are negative and $a_{21}, a_{23}, a_{31}, b_{11}$ are positive. Furthermore coefficients $a_{11}, a_{21}, a_{23}, a_{33}, b_{11}$ and $b_{12}$ depend on delay $\theta$. We denote third and second order polynomial in characteristic equation (28) by $P(\lambda, \theta):=$ $\lambda^{3}+p_{2} \lambda^{2}+p_{1} \lambda+p_{0}$ and $Q(\lambda, \theta):=q_{2} \lambda^{2}+q_{1} \lambda+q_{0}$, where

$$
\begin{array}{ll}
p_{2}=-a_{11}-a_{33} & q_{2}=-b_{11} \\
p_{1}=a_{11} a_{33}-a_{12} a_{21} & q_{1}=b_{11} a_{33}-b_{12} a_{21} \\
p_{0}=a_{12}\left(a_{21} a_{33}-a_{23} a_{31}\right) & q_{0}=b_{12}\left(a_{21} a_{33}-a_{23} a_{31}\right)
\end{array}
$$

Hence we can rewrite characteristic equation $(28)$ as $P(\lambda, \theta)+Q(\lambda, \theta) e^{-\lambda \theta}=$ 0. The characteristic equation (28) admits infinite complex roots but only a 
finite number or zero have positive real part. From corollary in Ruan and Wei (2003), the sum of the orders of the zeros with positive real part can change only if a zero appears on or crosses the imaginary axis. We note that $\lambda=0$ is not an eigenvalue indeed

$$
P(0, \theta)+Q(0, \theta)=\left(k-s_{k}\left(1-\delta_{k} e^{-(\alpha+\beta) \theta}\right)\right) v^{*}(1-h) u^{*} \eta \frac{\rho}{\left(1-v^{*}\right)^{2}}>0 .
$$

We observe that if $\lambda=i \omega(\theta)$ is an eigenvalue also $\lambda=-i \omega(\theta)$ is an eigenvalue because the coefficients of $P$ and $Q$ are real. For sake of simplicity, we neglect the dependence on time lag $\theta$ and, without loss of generality, we assume that $\omega>0$. We have that if $\lambda=i \omega$ is a purely imaginary root of characteristic equation (28) then $|P(i \omega, \theta)|^{2}=|Q(i \omega, \theta)|^{2}$.

We suppose that $P(i \omega, \theta)+Q(i \omega, \theta) \neq 0$. This assumption ensures that $P$ and $Q$ have no common imaginary roots and $Q(i \omega, \theta) \neq 0$ otherwise, from $P(i \omega, \theta)+Q(i \omega, \theta) e^{-i \omega \theta}=0$, it follows that also $P(i \omega, \theta)=0$. We observe that $P(i \omega, \theta)+Q(i \omega, \theta)=a_{0}-a_{2} \omega^{2}+i \omega\left(a_{1}-\omega^{2}\right)$ where $a_{0}, a_{1}$ and $a_{2}$ are given by equations (18) (replacing $\delta_{k}$ with $\delta_{k} e^{-(\alpha+\beta) \theta}$ ). We conclude that $P(i \omega, \theta)+Q(i \omega, \theta)=0$ iff $a_{1}>0$ and $a_{0}-a_{1} a_{2}=0$. If these last conditions are not satisfied from $e^{-i \omega \theta}=-\frac{P(i \omega, \theta)}{Q(i \omega, \theta)}$, considering real and imaginary parts, we have

$$
\left\{\begin{array}{l}
\cos \omega \theta=-\operatorname{Re}\left(\frac{P(i \omega, \theta)}{Q(i \omega, \theta)}\right)=-\frac{\left(p_{2} \omega^{2}-p_{0}\right)\left(q_{2} \omega^{2}-q_{0}\right)+q_{1} \omega^{2}\left(p_{1}-\omega^{2}\right)}{\left(q_{2} \omega^{2}-q_{0}\right)^{2}+q_{1} \omega^{2}} \\
\sin \omega \theta=\operatorname{Im}\left(\frac{P(i \omega, \theta)}{Q(i \omega, \theta)}\right)=\omega \frac{q_{1}\left(p_{2} \omega^{2}-p_{0}\right)-\left(p_{1}-\omega^{2}\right)\left(q_{2} \omega^{2}-q_{0}\right)}{\left(q_{2} \omega^{2}-q_{0}\right)^{2}+q_{1} \omega^{2}}
\end{array}\right.
$$

Let $\omega(\theta)$ a positive solution of $F(\omega, \theta)=0$ for $\theta \in I$, where $I \subset] 0,+\infty[$, and let $\phi(\theta) \in[0,2 \pi]$ such that

$$
\left\{\begin{array}{l}
\cos \phi(\theta)=-\operatorname{Re}\left(\frac{P(i \omega, \theta)}{Q(i \omega, \theta)}\right)=-\frac{\left(p_{2} \omega^{2}-p_{0}\right)\left(q_{2} \omega^{2}-q_{0}\right)+q_{1} \omega^{2}\left(p_{1}-\omega^{2}\right)}{\left(q_{2} \omega^{2}-q_{0}\right)^{2}+q_{1} \omega^{2}} \\
\sin \phi(\theta)=\operatorname{Im}\left(\frac{P(i \omega, \theta)}{Q(i \omega, \theta)}\right)=\omega \frac{q_{1}\left(p_{2} \omega^{2}-p_{0}\right)-\left(p_{1}-\omega^{2}\right)\left(q_{2} \omega^{2}-q_{0}\right)}{\left(q_{2} \omega^{2}-q_{0}\right)^{2}+q_{1} \omega^{2}}
\end{array}\right.
$$


By theorem 2.2 in Beretta and Kuang (2002), we have that if $\theta^{*} \in I$ is such that

$$
\theta^{*}=\frac{\phi\left(\theta^{*}\right)+2 \pi n}{\omega\left(\theta^{*}\right)}
$$

for some $n \in \mathbb{N}$ then a pair of simple conjugate pure imaginary roots $\lambda\left(\theta^{*}\right)=$ $\pm i \omega\left(\theta^{*}\right)$ exists at $\theta=\theta^{*}$. Furthermore, if

$$
\delta\left(\theta^{*}\right):=\left.\frac{d \operatorname{Re} \lambda}{d \theta}\right|_{\lambda=i \omega\left(\theta^{*}\right)}
$$

then if $\delta\left(\theta^{*}\right)>0$ and consequently the pair of simple conjugate pure imaginary roots crosses the imaginary axis from left to the right; if $\delta\left(\theta^{*}\right)<0$, then the pair of simple conjugate pure imaginary roots crosses the imaginary axis from right to the left.

In these cases, the number of eigenvalues with positive real part changes and $\theta=\theta^{*}$ can be a stability switch. In the next section we performed some numerical simulations showing the existence of stability switch when $\theta$ increases.

\section{Parameters and numerical simulations.}

\subsection{Parameters.}

To perform the numerical simulations of our model, we make use of the following set of parameters consistent with the real world:

$$
\begin{array}{lllll}
\epsilon=1 & \alpha=0.02 & \rho=0.076 & \gamma=1.23 & \beta=0.01 \\
s_{k}=0.1 & \delta_{k}=0.4 & k=0.14 & h \in[0,1] & \mu \in[0,1] \\
\xi \in[0.1,0.3] & \eta \in[0.7,1.05] & \theta \in[0,9] & &
\end{array}
$$

These parameters are such that to make unstable the equilibria A) and B). Therefore, we shall focus our attention on the equilibrium $\mathrm{C}$ ). 
Let us note that there are at least four crucial parameters in our model. They have a fundamental role in the dynamic evolution of the system. These parameters are:

i) The elasticity of price with respect to the labor cost variations $(h)$. As we said in the previous section, $h$ may be interpreted as a measure of the market power in the industry.

ii) The coefficient $\xi$ measuring the relative force of trade unions.

iii) The speed of adjustment of the expected inflation rate $\eta$.

iv) The intensity of public expenditure $\mu$.

All these parameters are bound one another in such a way to yield different behaviors of the system according to their particular combination. This implies that a wide variety of scenarios is possible either in the case where there is no delay in the tax revenues or in the case where a lag between the accrual and the payment of taxes exists.

\subsection{The case with no $\operatorname{lag}(\theta=0)$.}

In contrast with Wolfstetters conclusion, while a pro-cyclical fiscal policy $(\mu<0)$ makes the system always unstable, a counter-cyclical policy $(\mu>0)$ may be such that to make it either stable or unstable. The bifurcation values of $\mu$ are depicted in the figure 1 as a function of $h$. The bifurcation is always supercritical whatever the values of $\xi$ and $\eta$ may be. The red curve in that figure divides the plane between the stable (right hand side) from the unstable area (left hand side). As we can see, a very low intensity of 

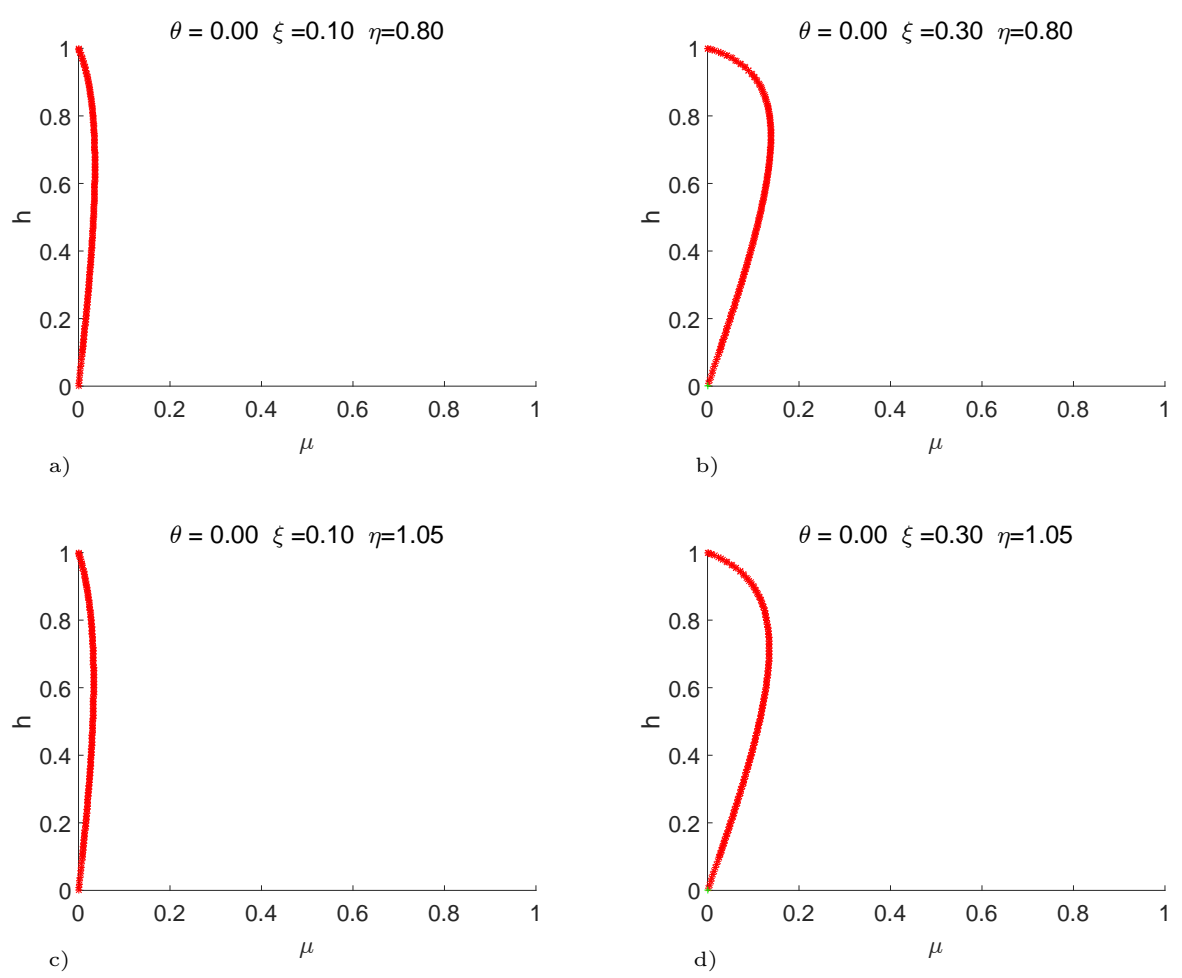

Figure 1: Bifurcation diagrams.

public expenditure is able to stabilize the system if both the market power of firms $(h)$ and the relative force of workers $(\xi)$ are low (fig.1a and fig. 1c). When the firms power market increases, the instability area augments and its extension increases if also the force of workers is rising (figures $1 \mathrm{~b}$ and 1d). This happens either in the case with slight or high reaction of the expected rate of inflation to the prices dynamics $(\eta)$. From the economic point of view, this means that a labor market with low competition ( $h$ and $\xi$ high enough) has the tendency to rise the system instability. At least one side of this market must be weaker than the other is to increase the possibility that the system may be stable. Furthermore, it is important to point out 
that without sufficient information about the degree of competition in the markets, the policy makers risk to overcome the minimum public expenditure able to stabilize the system.

4.3. The case with a delay $(\theta>0)$.
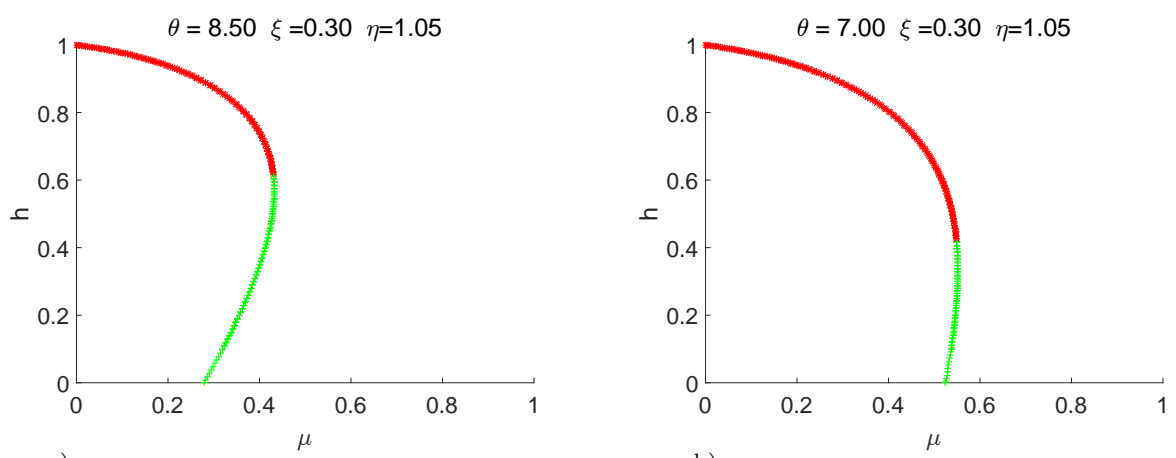

a)

b)
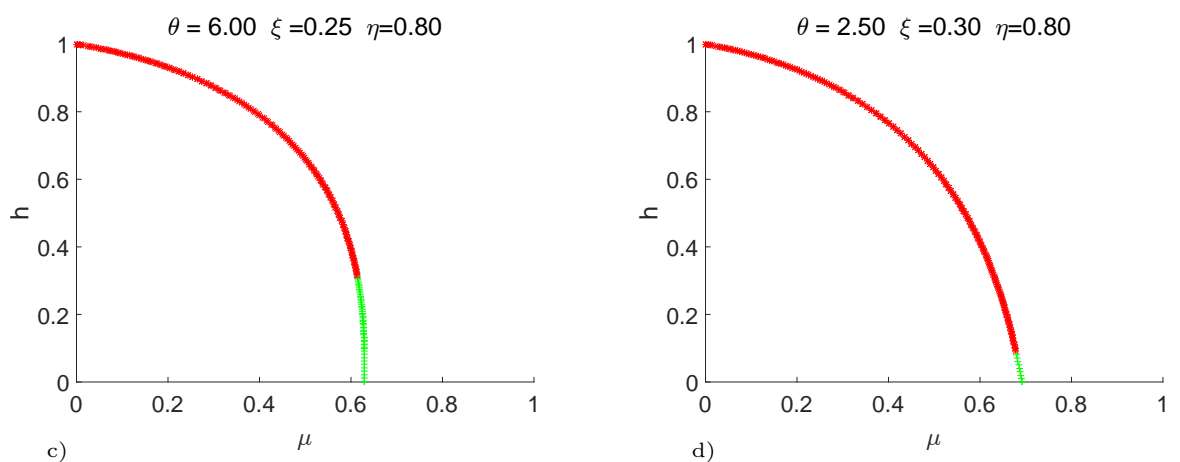

Figure 2: Bifurcation diagrams.

When a lag between the accrual and the payment of taxes exists and is high, the strength of public expenditure $(\mu)$ needs at least a value not less than $27 \%$ to yields stability conditions when $h$ is near to zero. If the market power of the firms increases, $\mu$ have to rise to preserve stability (figure 2a). The expansion of $\mu$ is needed up to a value of $h$ high enough $(h \approx 0.8)$, then a minor public expenditure in order that stability conditions tend to prevail. 
This happens because a high power market of the firms get off real wages and employment tends to rise.

If the time delay is reduced, with $\mathrm{h}$ near zero, the strength of public expenditure increases slowly and reaches a percentage in the neighborhood of $70 \%$ to sustain the system stability. Unlike the previous case, increasing $\mathrm{h}$ always implies a progressive reduction of $\mu$. This behavior of the system persists with a wide set of parameters economically meaning.

About the speed of adjustment of the expected inflation and the relative force of trade unions, we have to point out that they play a role in the definition of the bifurcation values and their qualitative properties. Looking at figures $2 \mathrm{a} 2 \mathrm{~d}$, we can see that these parameters are able to change the subcritical bifurcation (red line in the figures) in a supercritical one (green line). Thus, the emergence of stable limit cycles is possible. However, as figures show, the time delay reduction reduces the possibilities of supercritical bifurcations. An example of stable limit cycle is depicted in the figure 3 .

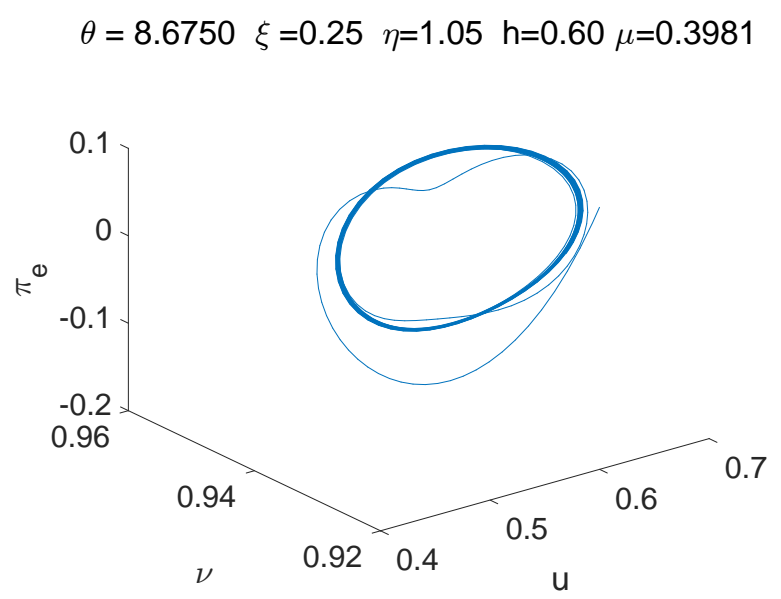

Figure 3: Stable limit cycle. 


\section{Concluding remarks.}

In this paper, we analyzed a growth cycle model in the tradition of Goodwin's (1967) class struggle approach to economic dynamics. Following the line of research explored by Wolfstetter, we added in the Goodwin model the public sector and emphasized the role of the degree of competition in the markets. As it happens in the real world, we assumed that the tax revenues coming from labor income are currently perceived by the government, while we supposed the existence of a finite time delay between the accrual and the payment of taxes pertaining to profits realized by the industry.

Our model shows that, when there is no time delay, in contrast with Wolfstetter's conclusions, the counter cyclical fiscal policy is the unique method for preventing economic fluctuations, only if policy makers know the real degree of competition in the markets. Lacking that information, the minimum public expenditure able to stabilize the system may likely be overcome, with negative consequences on the public debt.

Conclusions in the presence of a time delay in the tax revenues are less simple. In this case, it seems that there is a link between the length of the lag and the level of the market power of the firms. In fact, progressive reductions of the intensity of the public expenditure are required when in the industry the market power is increasing. This happens because the price policy applied by the firms tends to get off real wages and the employment

levels may be preserved. Nevertheless, the possibility of fluctuations of the economic activity cannot be completely avoided by means of the strength of the public expenditure. This because the system may generate regular cycles depending on the relative force of trade unions and the speed of adjustment of 
the expected inflation. Therefore, stabilization policies may become partially ineffectual because of the presence in the system of elements out of the control of the policy makers.

\section{References}

[1] Asada T., Yoshida H., 2001. Stability, Instability and Complex Behavior in Macrodynamic Models with Policy Lag. Discrete Dynamics in Nature and Society 5, 281-295.

[2] Beretta E., Kuang Y., 2002. Geometric stability switch criteria in delay differential system with delay dependent parameters. SIAM J. Math. Anal. 33, 5, 1144-1165.

[3] De Cesare L., Sportelli M., 2005. A dynamic IS-LM model with delayed taxation revenues. Chaos, Solitons and Fractals 25, 233-244.

[4] Engelborghs K., Luzyanina T., and Roose D., 2002. Numerical bifurcation analysis of delay differential equations using DDE-BIFTOOL, ACM Trans. Math. Softw. 28 (1), 1-21.

[5] Fanti L., 2004. Fiscal policy and tax collection lags: stability, cycles and chaos. Rivista Internazionale di Scienze Economiche e Commerciali 51. 341-365.

[6] Fanti L., Manfredi P., 2007. Chaotic business cycles and fiscal policy: An IS-LM model with distributed tax collection lags. Chaos, Solitons and Fractals 32, 736-744. 
[7] Goodwin R. M., 1967. A Growth Cycle. In: Feinstein, C. H. (Ed.), Socialism, Capitalism and Economic Growth. Cambridge University Press, Cambridge, 54-58.

[8] Hale J., 1977. Theory of functional differential equations, SpringerVerlag, New York.

[9] Kaldor N., 1940. A model of the trade cycle. Economic Journal, 50, 78-92.

[10] Kaldor N., 1961. Capital accumulation and economic growth. In: Lutz, F. A., Hague, D. C. (Eds.), The Theory of Capital. St. Martin's Press, New York, 177-228.

[11] Kuznetsov, Y. A., 2004. Elements of Applied Bifurcation Theory. Springer.

[12] Matsumoto A., 2008. Destabilizing effects on income adjustment process with fiscal policy lags. Metroeconomica 4, 713-735.

[13] Neamtu M., Opris D., Chilarescu C., 2007. Hopf bifurcation in a dynamic IS-LM model with time delay. Chaos Solitons and Fractals 34, $519-530$.

[14] Ruan S., Wei J., 2003. On the zeros of trascendental functions with applications to stability of delay differential equations with two delays, Dynamics of Continuous, Discrete and Impulsive Systems Series A: Mathematical Analysis 10, 863-874. 
[15] Sasakura, K., 1994. On the dynamic behavior of Schinasi's business cycle model. Journal of Macroeconomics 16, 423-444.

[16] Takamasu, A., 1995. On the effectiveness of the fiscal policy in the Goodwin growth cycle model. In: Aoki, N., Shiraiwa, K., Takahashi, Y. (Eds.), Proceeding of the International Conference on Dynamical Systems and Chaos, vol. 1. World Scientific Publishing, Singapore, 433436.

[17] Volterra V., 1931. Ricerche matematiche nelle associazioni biologiche. Giornale dell'Istituto Italiano degli Attuari, Anno II, n. 3, 295-355.

[18] Wolfstetter E., 1982. Fiscal Policy and the Classical Growth Cycle. Zeitschrift für Nationalökonomie 42, 375-393.

[19] Xiaofeng L., Chuandong L., Shangbo Z., 2005. Bifurcation and chaos in macroeconomic models with policy lag. Chaos Solitons and Fractals 25, 91-108.

[20] Yoshida H, Asada T., 2007. Dynamic analysis of policy lag in a KeynesGoodwin model: stability, instability, cycles and chaos. Journal of Economic Behavior and Organization 62, 441-469. 